\title{
The epicardium as modulator of the cardiac autonomic response during early development
}

\section{Citation for published version (APA):}

Kelder, T. P., Duim, S. N., Vicente-Steijn, R., Végh, A. M. D., Kruithof, B. P. T., Smits, A. M., van Bavel, T. C., Bax, N., Schalij, M. J., Gittenberger-de Groot, A. C., DeRuiter, M. C., Goumans, M. J. T. H., \& Jongbloed, M. R. M. (2015). The epicardium as modulator of the cardiac autonomic response during early development. Journal of Molecular and Cellular Cardiology, 89, 251-259. https://doi.org/10.1016/j.yjmcc.2015.10.025

DOI:

10.1016/j.yjmcc.2015.10.025

Document status and date:

Published: 01/12/2015

\section{Document Version:}

Publisher's PDF, also known as Version of Record (includes final page, issue and volume numbers)

\section{Please check the document version of this publication:}

- A submitted manuscript is the version of the article upon submission and before peer-review. There can be important differences between the submitted version and the official published version of record. People interested in the research are advised to contact the author for the final version of the publication, or visit the $\mathrm{DOI}$ to the publisher's website.

- The final author version and the galley proof are versions of the publication after peer review.

- The final published version features the final layout of the paper including the volume, issue and page numbers.

Link to publication

\section{General rights}

Copyright and moral rights for the publications made accessible in the public portal are retained by the authors and/or other copyright owners and it is a condition of accessing publications that users recognise and abide by the legal requirements associated with these rights.

- Users may download and print one copy of any publication from the public portal for the purpose of private study or research.

- You may not further distribute the material or use it for any profit-making activity or commercial gain

- You may freely distribute the URL identifying the publication in the public portal.

If the publication is distributed under the terms of Article 25fa of the Dutch Copyright Act, indicated by the "Taverne" license above, please follow below link for the End User Agreement:

www.tue.nl/taverne

Take down policy

If you believe that this document breaches copyright please contact us at:

openaccess@tue.nl

providing details and we will investigate your claim. 


\title{
The epicardium as modulator of the cardiac autonomic response during early development
}

\author{
Tim P. Kelder ${ }^{\mathrm{a}, 1}$, Sjoerd N. Duim ${ }^{\mathrm{b}, 1}$, Rebecca Vicente-Steijn ${ }^{\mathrm{a}, \mathrm{d}, \mathrm{e}}$, Anna M.D. Végh ${ }^{\mathrm{b}}$, Boudewijn P.T. Kruithof ${ }^{\mathrm{b}}$, \\ Anke M. Smits ${ }^{\text {b }}$, Thomas C. van Bavel ${ }^{\mathrm{a}, \mathrm{b}}$, Noortje A.M. Bax ${ }^{\mathrm{c}}$, Martin J. Schalij ${ }^{\mathrm{d}}$, \\ Adriana C. Gittenberger-de Groot ${ }^{\mathrm{a}, \mathrm{d}}$, Marco C. DeRuiter ${ }^{\mathrm{a}}$, \\ Marie-José Goumans ${ }^{\mathrm{b}, *, 1}$, Monique R.M. Jongbloed ${ }^{\mathrm{a}, \mathrm{d}, * *, 1}$ \\ a Department of Anatomy \& Embryology, Leiden University Medical Center, Leiden, The Netherlands \\ b Department of Molecular Cell Biology, Leiden University Medical Center, Leiden, The Netherlands \\ c Department of Biomedical Engineering, Eindhoven University of Technology, The Netherlands \\ ${ }^{d}$ Department of Cardiology, Leiden University Medical Center, Leiden, The Netherlands \\ e ICIN Netherlands Heart Institute, Utrecht, The Netherlands
}

\section{A R T I C L E I N F O}

\section{Article history:}

Received 10 July 2015

Received in revised form 14 October 2015

Accepted 20 October 2015

Available online 23 October 2015

\section{Keywords:}

Epicardium

Cardiac development

Autonomic nervous system

Autonomic modulation

Cardiac conduction system

\begin{abstract}
A B S T R A C T
The cardiac autonomic nervous system (cANS) modulates heart rate, contraction force and conduction velocity. The embryonic chicken heart already responds to epinephrine prior to establishment of the cANS. The aim of this study was to define the regions of the heart that might participate in modulating the early autonomic response to epinephrine. Immunofluorescence analysis reveals expression of neural markers tubulin beta-3 chain and neural cell adhesion molecule in the epicardium during early development. In addition, expression of the $\beta 2$ adrenergic receptor, the receptor for epinephrine, was found in the epicardium. Ex-ovo micro-electrode recordings in hearts with inhibition of epicardial outgrowth showed a significantly reduced response of the heart rate to epinephrine compared to control hearts. This study suggests a role for the epicardium as autonomic modulator during early cardiac development.
\end{abstract}

(c) 2015 Elsevier Ltd. All rights reserved.

\section{Introduction}

The cardiac autonomic nervous system (cANS) is essential in modulating cardiac function by altering heart rate (chronotropy), conduction velocity (dromotropy) and force of contraction (inotropy) [1]. Dysfunctioning of the cANS plays a role in the pathogenesis of arrhythmias [1] and hypertension [2] and is involved in disease progression in heart failure [3]. Furthermore, normal functioning of the cANS is important for the prognosis of adult patients with congenital heart disease [4].

Abbreviations: $\beta 1 \mathrm{AR}, \beta 1$ adrenergic receptor; $\beta 2 \mathrm{AR}, \beta 2$ adrenergic receptor; cANS cardiac autonomic nervous system; CTNI, cardiac Troponin I; E, embryonic day; EMT, epithelial-to-mesenchymal transition; HH, Hamburger and Hamilton; NCAM, Neural Cell Adhesion Molecule; NCC, neural crest cells; NGF, nerve growth factor; PEO, proepicardial organ; RA, retinoic acid; RXR $\alpha$, retinoid X receptor- $\alpha$; SV, sinus venosus; TUBB3, tubulin beta-3 chain; WT1, Wilms' tumor-1.

* Correspondence to: M.-J. Goumans, Dept. of Molecular Biology, Leiden University Medical Center, S-1-P, P.O. Box 9600, 2300 RC Leiden, The Netherlands.

** Correspondence to: M.R.M. Jongbloed, Dept. of Anatomy \& Embryology and Cardiology, Leiden University Medical Center, S-1-P, P.O. Box 9600, 2300 RC Leiden, The Netherlands.

E-mail addresses: m.j.t.h.goumans@lumc.nl (M.-J. Goumans), m.r.m.jongbloed@lumc.nl (M.R.M. Jongbloed).

${ }^{1}$ Authors contributed equally.
Understanding the processes that govern normal cANS development may help in unraveling the pathophysiology of abovementioned disease processes and in developing targeted treatment options.

The cANS can be divided into a sympathetic and parasympathetic component. In general, sympathetic stimulation results in an increase of heart rate, conduction velocity and force of contraction, while parasympathetic stimulation has an opposing effect. Sympathetic neurons have their cell bodies primarily in the paravertebral stellate ganglion, whereas parasympathetic cell bodies are located in the cardiac ganglia [5]. The cells contributing to the cANS are derivatives of neural crest cells (NCCs) and cells of the nodose placode [6,7]. Kroese et al. demonstrated that prior to cardiac sympathetic innervation of the developing chicken embryo, the heart already responds to the catecholamine epinephrine [8]. This neurotransmitter binds to beta $(\beta)$ adrenergic receptors (AR), thereby activating cAMP dependent signaling [9], resulting in an increase in heart rate, conduction velocity and force of contraction [5]. It is remarkable that expression of enzymes necessary for production of catecholamines was found throughout the myocardium during cardiac development in rat [10], even before production is observed in the adrenal glands [11,12]. After addition of epinephrine in chick at Hamburger and Hamilton $\left(\mathrm{HH}^{13}\right)$ stage $20-24$, several hemodynamic parameters, including heart rate, increased significantly [8]. This 
supports an important role for catecholamines in the heart during early embryo development. Furthermore, stimulation of chick embryos with isoproterenol ( $\beta$-agonist) at embryonic day 7 ( $\mathrm{HH} 30-31)$ resulted in an increase in cAMP [14]. In addition to responding to $\beta$-adrenergic stimulation, the early embryonic chicken heart was also shown to respond to $\beta$-adrenergic receptor blockade by reducing heart rate and cardiac output [15]. Thus prior to establishment of the cANS, the heart already responds to autonomic stimulation and blockade.

Interestingly, Kroese et al. showed that after treatment with all-trans retinoic acid (RA) the reaction to epinephrine, including the increase in heart rate, was significantly reduced [8]. Normal RA signaling has been shown to be important for proper development of the epicardium [16]. This single layer of cells is derived from the proepicardial organ (PEO) and covers the initially bare primary myocardial heart tube. Cells derived from the epicardium are known to play an essential role during normal cardiac development and defects in epicardial development result in cardiac malformations (reviewed in [17]).

The aim of the current study was to identify which cell population in the developing heart plays a role in modulating the autonomic response during early development. Our studies reveal unanticipated expression of neuronal markers in the epicardium during early cardiac development. To investigate a potential role of the epicardium, electrophysiological experiments were performed with and without inhibition of epicardial outgrowth.

\section{Material and methods}

\subsection{Animals}

Immunohistochemical analysis was performed in wild type mouse embryos with a mixed genetic background of different embryonic stages (E9.5-E17.5, mice described in [18]). The morning of the vaginal plug was considered E0.5. Pregnant mice were euthanized using $\mathrm{CO}_{2}$ exposure and cervical dislocation. Animal care was in accordance with national and institutional guidelines and approved by the animal experiments committee of the Leiden University Medical Center.

To study protein expression in chick embryos, fertilized eggs of the White Leghorn chicken were incubated at $37^{\circ} \mathrm{C}$ and $80 \%$ humidity. Hearts were excised, and staged according to Hamburger and Hamilton (HH) [13]. Tissue was fixed in $4 \%$ paraformaldehyde for $24 \mathrm{~h}$ and subsequently embedded in paraffin and sectioned $(5 \mu \mathrm{m})$ for immunohistochemical analysis.

\subsection{Human fetal tissue}

A 5-week-old human fetal heart was collected after elective abortion based on individual informed consent procedures conforming to the Declaration of Helsinki. Furthermore, the study was approved by the Medical Ethics committee of the Leiden University Medical Center. Tissue was treated as described above.

\subsection{Immunohistochemistry}

The protocol used for immunohistochemical staining was described previously [19]. Briefly, slides were rehydrated, subjected to heatinduced epitope retrieval and incubated with the following list of antibodies: anti-cardiac Troponin I (CTNI) (myocardial marker, 1:1000, 4T21/2, HyTest Ltd), anti-Wilms' tumor-1 (WT1) (expressed in the epicardium, 1:1000, ab89901, Abcam), anti-tubulin beta-3 chain (TUBB3) (neuronal marker, 1:200, AB78078, Abcam), Neural Cell Adhesion Molecule (NCAM) (neuronal marker, 1:250, AB5032, Merck), anti- $\beta 1$ adrenergic receptor ( $\beta 1 A R$ ) (receptor for epinephrine, 1:200, PA528808, Thermo Scientific), and anti- $\beta 2$ adrenergic receptor ( $\beta 2 A R$ ) (receptor for epinephrine, 1:200, ab61778, Abcam). To amplify WT1 expression Tyramide Signal Amplification (PerkinElmer) was used. Visualization was achieved by incubation with Alexa Fluor® 488 streptavidin
(Invitrogen). The remainder of primary antibodies was visualized with Alexa-conjugated fluorescent secondary antibodies (Invitrogen) at a final concentration of 1:200. DAPI (D3571, 1/1000; Life Technologies) was used as a nuclear stain, after which slides were mounted with Prolong gold (Life Technologies).

\subsection{Mechanical blocking of the proepicardial organ (PEO)}

Mechanical inhibition of the epicardial outgrowth in the chicken embryo was performed as described previously [20]. At HH15, a window was created in the eggshell, after which the embryonic membranes were opened. Subsequently, a small piece of eggshell membrane was placed between the PEO and developing heart tube, after which the egg was re-incubated until the desired stage. In order to verify that outgrowth of the epicardial layer was hampered, hearts were sectioned and a Hematoxylin and Eosin staining was performed.

\subsection{Ex-ovo extracellular micro-electrode recordings and epinephrine administration}

To investigate the effect of epinephrine on heart rate, electrophysiological measurements were performed in embryonic chicken hearts at different developmental stages. After reaching the desired stage of development, embryos were extracted from the egg. The heart and some surrounding tissue were excised and placed in a temperaturecontrolled $\left(37 \pm 0.1{ }^{\circ} \mathrm{C}\right)$ tissue bath containing Tyrode. Recordings were performed using a previously described protocol [21]. Recording electrodes were placed on the atrium and the ventricular apex, and a reference electrode in the tissue bath. The hearts of five groups of embryos were studied: 1 . HH15 embryos ( $n=5$ ), when the heart is not yet covered by epicardium; 2 . HH19 embryos $(n=3)$, when epicardial covering of the sinus venosus, atria and AV-canal has commenced; 3. HH21 embryos $(n=3)$, when migration of epicardial cells around the heart is (nearly) complete [22]; 4. HH24-25 control embryos $(\mathrm{n}=9$, no surgical manipulation), when epicardial covering has been completed and subepicardial mesenchyme is present; 5. HH24-25 embryos $(n=9)$ after inhibition of epicardial outgrowth.

The hearts were allowed to reach a stable baseline heart rate (which was comparable between all studied groups, Supplemental Fig. S1), after which $100 \mu \mathrm{l}$ of pre-warmed epinephrine ( $1 \mathrm{mg} / \mathrm{ml}$, Centrafarm, The Netherlands) was directly pipetted onto the heart. Pre-warmed Tyrode was administered as a negative control to HH24-25 hearts $(n=6)$. The relative response to epinephrine was calculated by correcting the change in heart rate for the baseline heart rate. The heart rate was calculated every $10 \mathrm{~s}$ and plotted.

\subsection{Statistical analysis}

The Mann-Whitney U-test (two groups) or Kruskal-Wallis test (>two groups) were used, since the data was not normally distributed. $\mathrm{P}<0.05$ was considered statistically significant. Data shown is mean \pm S.E.M. Statistical analysis was performed using the Graphpad Prism 6 software package (Graphpad Software).

\section{Results}

\subsection{During early cardiac development the neuronal marker TUBB3 is expressed by the epicardium}

In order to investigate which cell population plays a potential role in modulation of the early cardiac autonomic response, protein expression of the neuronal marker TUBB3 was analyzed during cardiogenesis.

At E9.5, the primary heart tube is not covered by epicardial cells and the PEO is recognizable (Fig. 1a-d). Co-expression of neuronspecific TUBB3 and WT1 was observed in a subset of cells in the PEO (Fig. 1a-d). WT1 +/TUBB3 - and WT1 -/TUBB3 + cells were also 


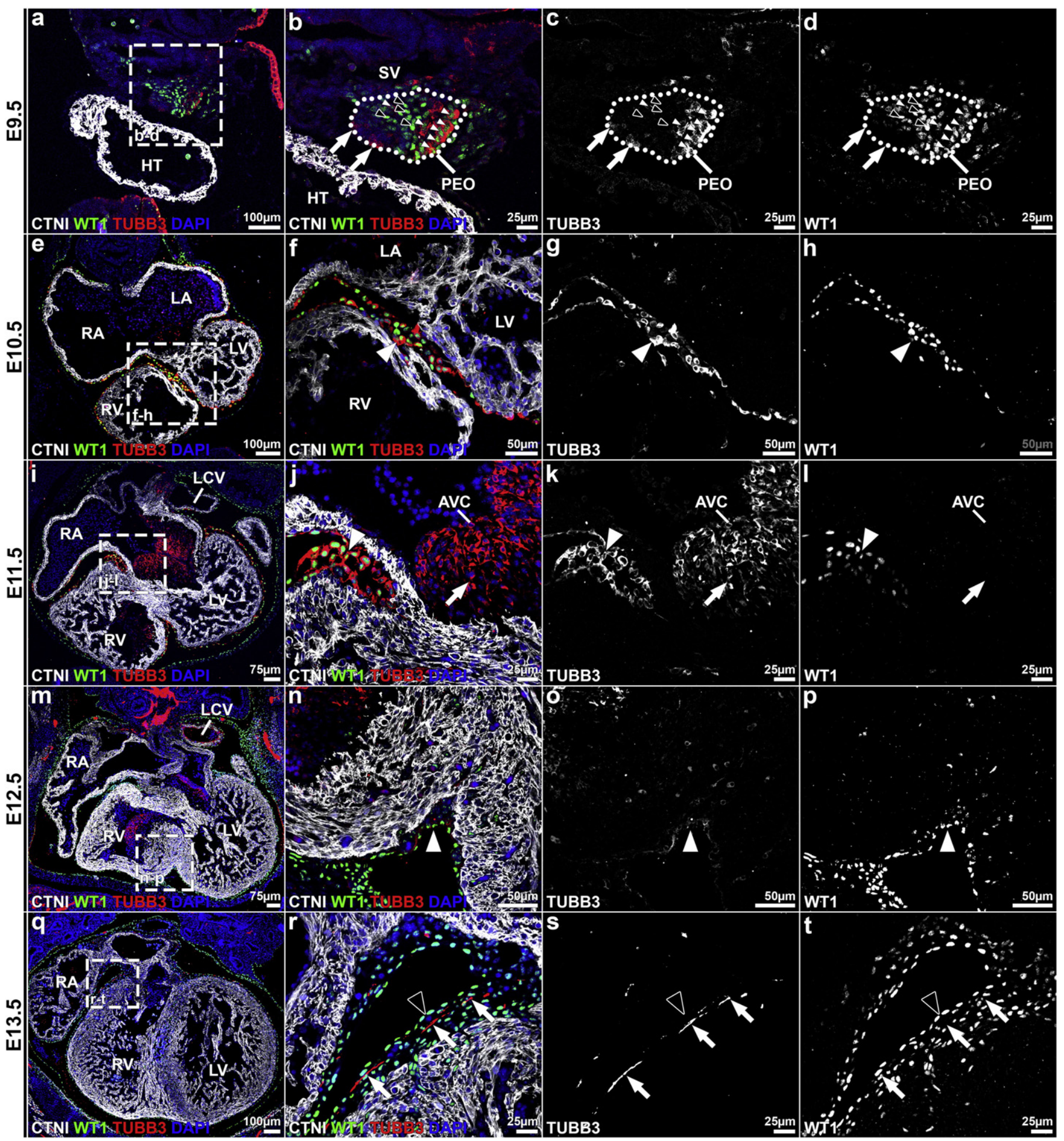

Fig. 1. The neuronal specific marker TUBB3 is expressed in the epicardium and subepicardium during early development. a-d. At E9.5, the PEO (dotted line) is recognizable and the heart tube is not covered by epicardium. WT1 +/TUBB3 + cells (white arrowheads in b-d), WT +/TUBB3 - cells (black arrowheads in b-d) and WT1 - /TUBB3 + cells (arrows in b-d) are observed within the PEO. e-h. At E10.5 the heart is partially surrounded by epicardial cells and co-expression of TUBB3 with WT1 is seen in most epicardial cells (arrowheads in f-h). i-l. Coexpression of TUBB3 and WT1 in the epicardium is still present at E11.5 (arrowheads in j-1). TUBB3 expression is also seen in the endocardial cushions (arrows in j-1), shown here at E11.5. $\mathrm{m}$-p. Co-expression of TUBB3/WT1 decreases from E12.5 (compare white arrowhead in k with white arrowhead in o). q-t. At E13.5 the expression of TUBB3 in the WT1 + epicardium (black arrowheads in $\mathrm{r}-\mathrm{t}$ ) is lost but is now present in subepicardial nerve fibers (arrows in $\mathrm{r}-\mathrm{t}$ ). HT, heart tube; SV, sinus venosus; PEO, proepicardial organ; RA, right atrium; LA, left atrium; RV, right ventricle; LV, left ventricle; LCV, left cardinal vein; AVC atrioventricular cushion.

present (Fig. 1a-d), confirming the heterogeneity of the PEO [23]. At E10.5, the epicardial layer has started to envelope the heart and showed co-expression of TUBB3 and WT1 in most epicardial cells (Fig. 1e-h). Co-expression of TUBB3 and WT1 was still clearly present at E11.5 in the majority of epicardial cells (Fig. 1i-1). Strong expression of TUBB3 was observed in the mesenchyme of the endocardial cushions in the OFT and AV canal, shown at E11.5 (Fig. 1i-1). TUBB3 expression in the epicardium decreased with ongoing development. At E12.5, only faint 
expression was observed in the epicardium (Fig. 1m-p), disappearing at E13.5 (Fig. 1q-t). At this stage, TUBB3 expression was only observed in subepicardial nerve fibers (Fig. 1q-t) and no co-expression of TUBB3 and WT1 was observed in the epicardium or subepicardium.

To confirm the neuronal phenotype of the epicardium, NCAM (neuronal marker) expression was analyzed. At E11.5, co-expression of NCAM and TUBB3 was seen in the nervous system, including the neural tube and dorsal root ganglion (Supplemental Fig. S2a-d). Furthermore, NCAM expression was seen in the epicardium, co-localizing with TUBB3 (Supplemental Fig. S2e-h), thereby confirming the neuronal phenotype of the epicardium.

In addition, TUBB3 expression was analyzed during early human fetal development. A subset of epicardial cells and cells in the endocardial cushions showed expression of TUBB3 (Supplemental Fig. S3b-c). Furthermore, cells in the subepicardial space showed TUBB3 expression (Supplemental Fig. S3d-e).

\subsection{A subpopulation of cells in the central nervous system co-express WT1 and TUBB3}

The neuronal marker TUBB3 showed protein expression in the epicardium, co-expressing with WT1. In order to provide possible evidence that these proteins are involved in normal neuronal function, expression of TUBB3 and WT1 was studied in the nervous system. At E11.5, the developing nervous system showed expression of TUBB3 (Fig. 2ad). No WT1 expression was found (Fig. 2a-d). Microscopic analysis at E13.5 revealed co-expression of TUBB3 and WT1 in the ventral region of the neural tube, as well as the roof of the 4th ventricle of the brain (Fig. 2e-g). WT1 expression was observed along the entire length of the neural tube (Fig. 2e). Co-expression of TUBB3 and WT1 in the spinal cord was still present at E17.5 (Fig. 2h-k).

\subsection{During early cardiac development the catecholamine receptor $\beta 2 A R$ is expressed by the epicardium}

To further confirm a potential neuronal phenotype of the epicardium, expression of the beta1 $(\beta 1)$ and beta2 $(\beta 2)$ adrenergic receptors (AR) was studied. To confirm neural co-expression of TUBB3 and $\beta 2 \mathrm{AR}$, these markers were studied at E11.5 in the nervous system. Colabeling of TUBB3 and $\beta 2 A R$ was seen in the dorsal root ganglion and neural tube (Fig. 3a-d).

In murine embryos at E11.5, most epicardial cells are TUBB3 + , and a subpopulation of epicardial cells showed co-expression with $\beta 2 \mathrm{AR}$ (Fig. 3e-h). $\beta 2 A R+$ cells were found throughout the epicardial layer, predominantly seen in the AV and interventricular sulcus (Fig. $3 \mathrm{e}-\mathrm{h}$ ). Furthermore, expression of $\beta 2 A R$ was seen in endocardial cells and a subpopulation of cells in the endocardial cushions (Fig. 3e) Expression of TUBB3 and $\beta 2 A R$ was seen in the subepicardium at E12.5, with a subset of cells co-expressing both markers (Fig. 3i-1). 31 AR expression was not observed in these stages (not shown).

\subsection{Inhibition of epicardial outgrowth results in a diminished response to epinephrine}

At HH21 of chick development, expression of $\beta 2 A R$ was found in the epicardium, PEO and endocardium (Supplemental Fig. S4) which is in agreement with the $\beta 2 A R$ expression found in mice. First, the role of the epicardial layer in the response of the heart to epinephrine was studied. For this purpose, chick hearts from embryos of subsequent stages of epicardial development were analyzed using ex-ovo microelectrode recordings (Fig. 4 and Supplemental Fig. S5). The relative increase ((maximal heart rate - baseline) / (baseline) $) * 100 \%$ in heart rate after administration of epinephrine was calculated. HH15 hearts, which lack epicardial covering, showed no increase in heart rate after administration of epinephrine (Fig. 4a and Supplemental Fig. S5a). A significant increase in heart rate was observed in hearts of HH19 (when epicardial covering of the sinus venosus, atria and AV-canal has initiated), HH21 (epicardial covering has (nearly) been completed) and HH24 (the occurrence of subepicardial mesenchyme) compared to HH15 embryos (Fig. 4b-e and Supplemental Fig. S5b). This indicates that the presence of epicardium is required for the response to epinephrine during early developmental stages.

To further substantiate the role of the epicardium in the response to epinephrine, an experimental model was used in which outgrowth of the epicardium is inhibited in chick embryos. To confirm epicardial inhibition, hearts were analyzed histologically, after the electrophysiological measurements. Analysis of the epicardial response to epinephrine was conducted at stage $\mathrm{HH} 24$, when the effects of the epicardial inhibition are clearly observed. At HH24, control hearts showed epicardial covering of the entire heart, with a cell-rich subepicardial space (Supplemental Fig. S6). After epicardial inhibition, large portions of the heart were not covered by epicardium and the regions covered with epicardium showed less subepicardial cells (Supplemental Fig. S6). Furthermore, compaction was hampered, as shown by a less dense compact myocardial layer compared to controls (Supplemental Fig. S6).

Ex-ovo micro-electrode recordings were performed after administration of epinephrine in control and inhibited hearts. After administration of epinephrine to control hearts $(n=9)$, a fast increase in heart rate was observed (Fig. 5a and Supplemental Fig. S5b). The relative increase in heart rate was $57,2 \pm 7,20 \%$. After epicardial inhibition $(n=9)$ however, this response was decreased (Fig. 5b,d p =0,03 and Supplemental Fig. S5d), with a relative increase in frequency of $24,8 \pm 4,33 \%$. Prewarmed Tyrode served as a negative control. Upon administration a short decrease in heart rate was observed, after which the heart rate returned to the baseline frequency (Fig. 5c and Supplemental Fig. S5c).

The earliest electrical activity is generated in the myocardium of the sinus venosus (SV) [21]. Since the response to epinephrine was altered after epicardial inhibition, the next step was to evaluate epicardial covering of the SV myocardium in these hearts.

Hearts that were classified as successful inhibition of epicardial outgrowth in Fig. 5, were microscopically subdivided into two categories based on epicardial covering of the SV. Hearts were classified as "mild inhibition" ( $n=4)$ when the myocardium of the SV was covered by epicardium, but no subepicardial cells were present (Fig. 6a,b). Hearts were classified as "severe inhibition" $(n=5)$ when the myocardium of the SV was (largely) devoid of epicardium and subepicardial cells were not present (Fig. 6d,e). Micro-electrode recordings revealed that the relative response to epinephrine was hampered in the mild inhibition group. The increase in heart rate was less pronounced and it took longer to return to the baseline heart rate after epinephrine administration compared to the control group (Fig. 6c). In the severely inhibited group, virtually no response to epinephrine was observed (Fig. 6f). The relative response to epinephrine was significantly decreased in the group classified as "severe inhibition" as compared to the "mild inhibition" group $(\mathrm{p}=0,016$ Fig. $6 \mathrm{~g})$.

\section{Discussion}

Autonomic modulation is essential for proper functioning of the heart and contributes to the prognosis of patients with heart failure and congenital heart disease. Early in development, the heart already responds to sympathetic stimulation, even prior to the presence of sympathetic nerve fibers [8]. The exact mechanism behind this early response is poorly understood. The current study provides new evidence that can account for this response. Key findings of this study are; 1) The epicardium expresses TUBB3, NCAM and B2AR during early development, which are known neuronal markers; 2) Inhibition of epicardial outgrowth results in a disturbed response to epinephrine; 3) The severity of inhibition of epicardial covering of the sinus venosus myocardium correlates to the severity of disturbance in the response to epinephrine. Together, these results suggest a role for the epicardium in autonomic modulation of the heart during early development. 


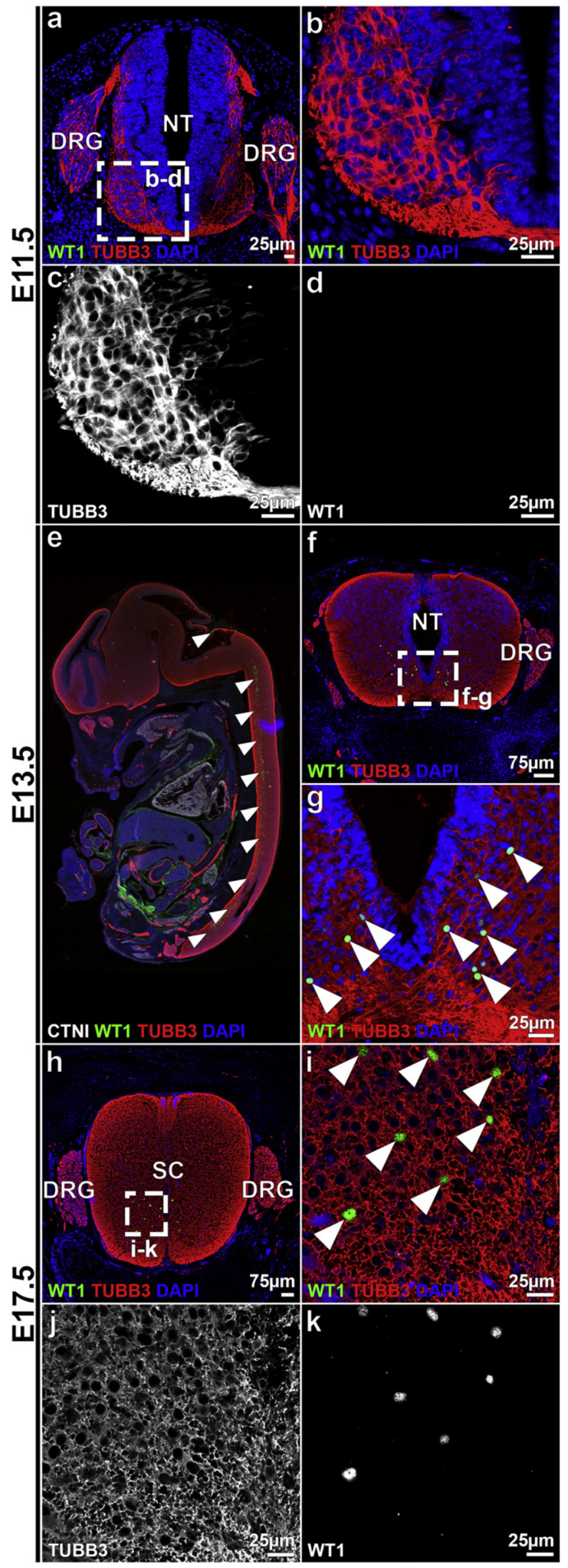

The current work describes expression of proteins known to be of importance in the nervous system in the epicardium. The tubulin isoform TUBB3 is primarily expressed in neurons and is important for axonal guidance and maintenance [24]. Expression however is not limited to neurons, since melanocytes (derived from the neural crest, as do neurons) also show TUBB3 expression [25,26]. To ensure the neuronal phenotype, staining of another neural marker, neural cell adhesion molecule (NCAM) was performed. NCAM expression was also present in the epicardium, confirming the neuronal phenotype of the epicardial layer. More indirect evidence suggesting a possible role for the epicardium in neural function is the co-expression of WT1 and TUBB3 in the central nervous system. Wt1, a transcription factor expressed in the embryonic PEO and epicardium, is necessary for normal epicardial and cardiac development [27]. The current manuscript shows WT1/TUBB3 coexpression in the central nervous system, which is in agreement with previous reports demonstrating an important role for Wt1 in neural functioning [28-32].

In addition to epicardial expression of NCAM and TUBB3, the receptor for epinephrine, $\beta 2 A R$, was expressed in the epicardium. Epinephrine binds to $\beta 2 \mathrm{AR}$, which activates adenyl cyclase, resulting cAMPdependant signaling [9], which leads to the sympathetic modulation of the heart rate, conduction velocity, and force of contraction.

To investigate the functional role of the epicardium in autonomic modulation, the response to epinephrine was analyzed in an experimental model in which normal outgrowth of the epicardium was inhibited. Results showed a significantly reduced response to epinephrine after epicardial inhibition. To further validate these results, epinephrine was administered in control chicken embryos at HH15, a stage in which no epicardial cells are present on the heart tube. Interestingly, these embryos did not show a response to epinephrine, confirming the data seen after epicardial inhibition. These results show that epicardial covering of the heart is (at least partially) responsible for a normal response to epinephrine during early cardiogenesis.

Absence of epicardial covering of the sinus venosus myocardium, as was described in the "severe inhibition group", results in absence of the epicardial $\beta 2 A R$. If epinephrine cannot bind to this receptor, cAMP mediated signaling is likely to be hampered, resulting in an absent response to epinephrine. Furthermore, it was recently shown that the $\beta 2 A R$ forms protein complexes with the funny-current ion channel HCN4, responsible for spontaneous depolarization of pacemaker cells. Disturbing the formation of the $\beta 2 \mathrm{AR} / \mathrm{HCN} 4$ protein complexes results in a hampered response to sympathetic stimulation [33], a result also observed after epicardial inhibition (this study).

Kroese et al. showed that disturbance of retinoic acid signaling results in a hampered response to epinephrine. Disturbing RA signaling in the epicardium by epicardial deletion of the retinoid X receptor- $\alpha$ $(\mathrm{RXR} \alpha)$, results in defective epithelial-to-mesenchymal transition (EMT), thinning of the myocardium, disturbed coronary arteriogenesis and ventricular pre-excitation [34,35]. This phenotype is also seen after disturbance of epicardial outgrowth in avian embryos [36] and in Wt1-null mice [27,37]. Wt1 regulates RA signaling by activating RALDH2, the enzyme involved in RA synthesis [38], and Wt1-null mice show downregulation of RALDH2 [27]. Vice versa, induction of RA signaling results in upregulation of Wt1 expression in chick epicardialderived cells [39]. Therefore, disturbing the normal outgrowth of the epicardium could result in hampering of RA signaling. Previous data showed that RA treatment in vitro results in neuronal differentiation, with an increase in $\beta 2 A R$ expression [40]. This indicates that RA signaling is important for normal $\beta 2 A R$ expression. Disturbing this by

Fig. 2. WT1 is expressed in the ventral horn of the spinal cord. a-d. At E11.5 TUBB3 is present in the neural tube, however, no co-expression with WT1 is found. e-g. From E13.5 co-expression of WT1 and TUBB3 is observed in the ventral horn of the neural tube and the roof of the 4th ventricle of the brain (arrowheads). h-k. Co-expression of TUBB3/WT1 is still present in the spinal cord at E17.5 (arrowheads in i-k). DRG, dorsal root ganglion; NT, neural tube; SC, spinal cord. 


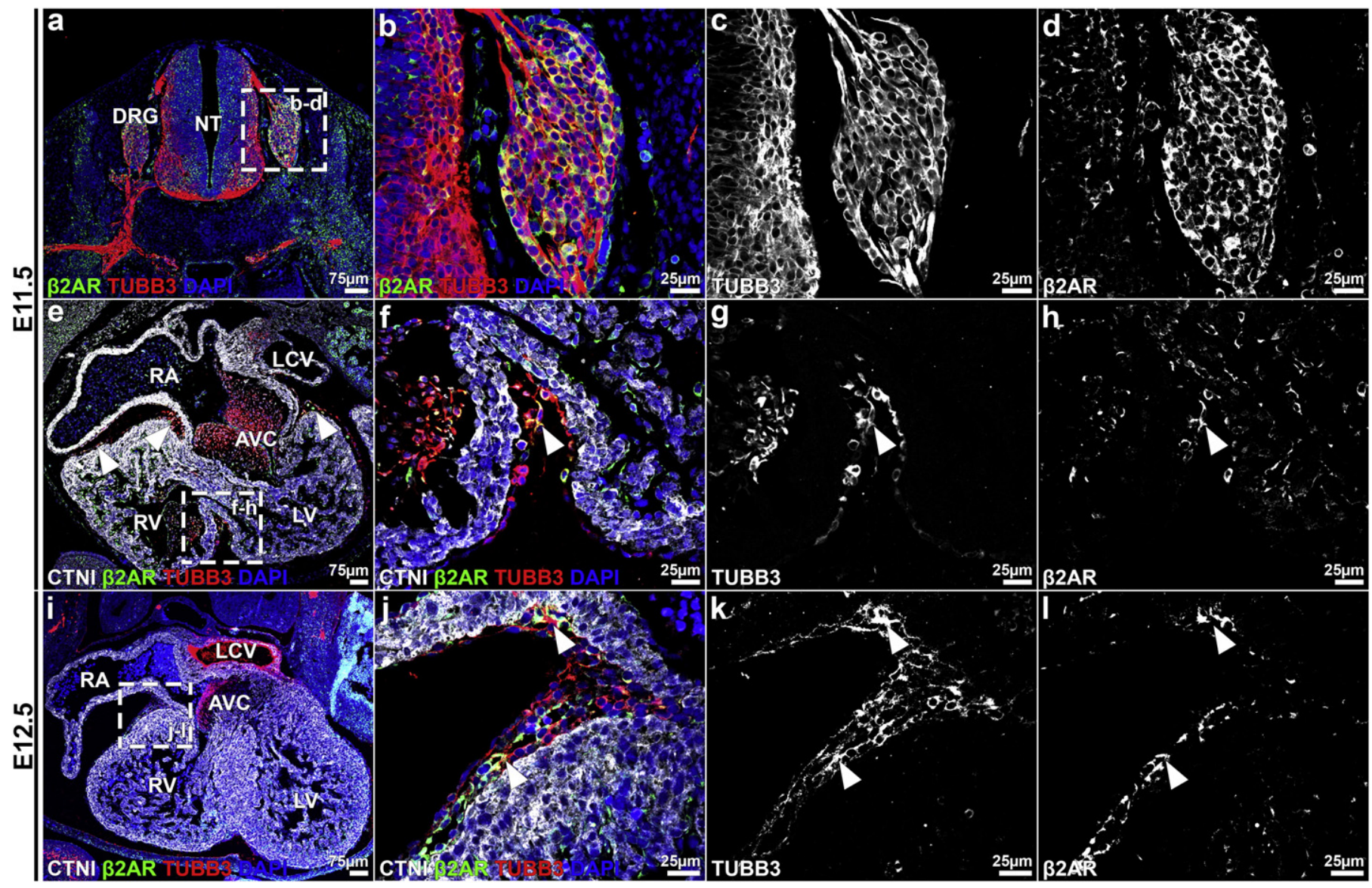

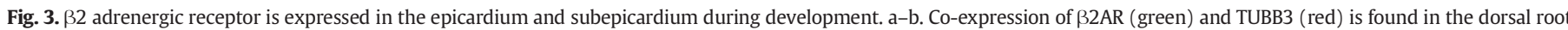

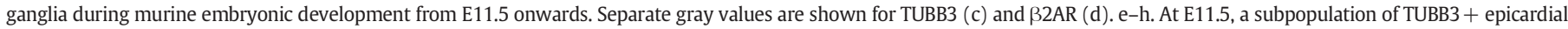

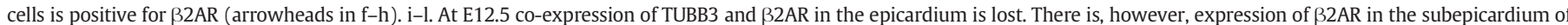

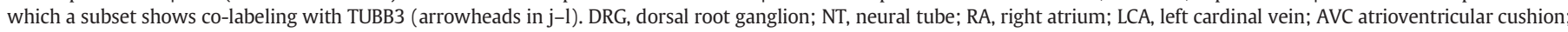
$\mathrm{RV}$, right ventricle; $\mathrm{LV}$, left ventricle.
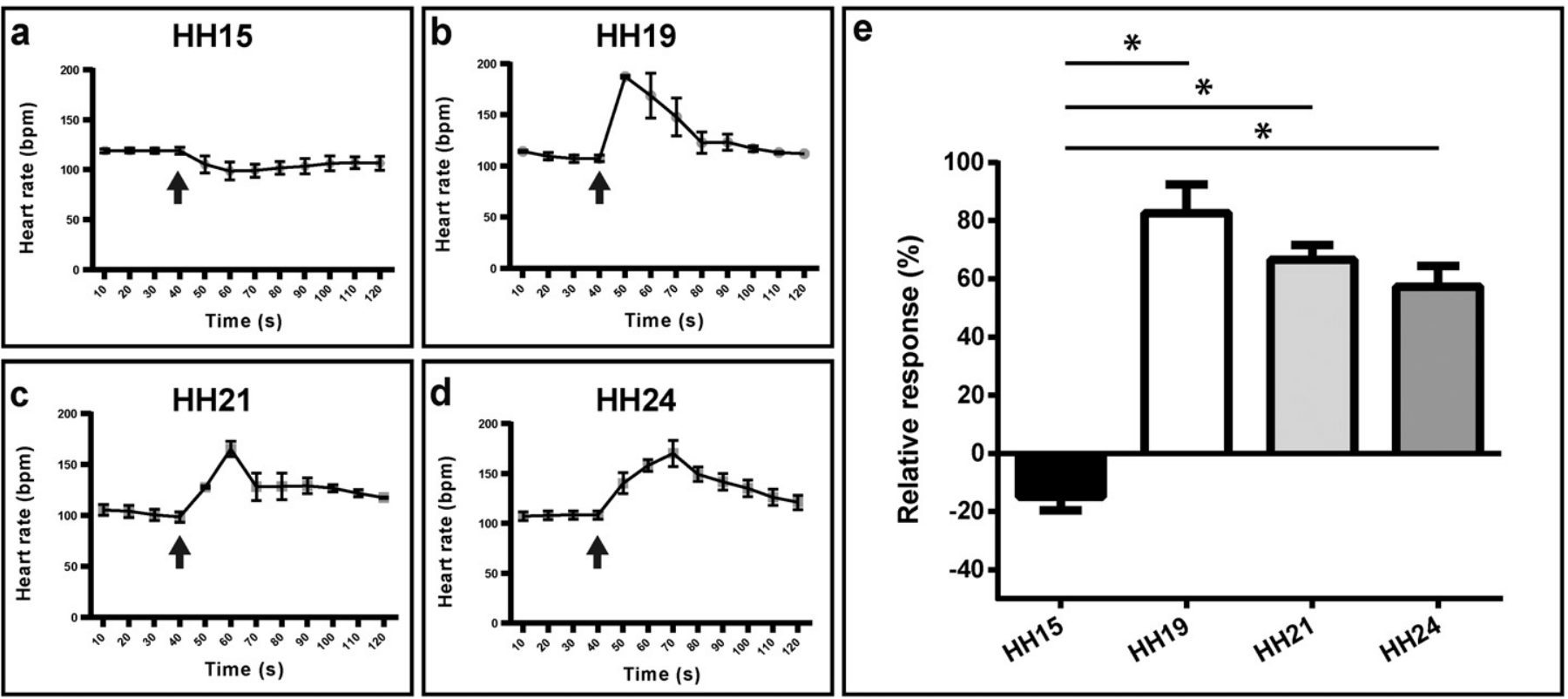

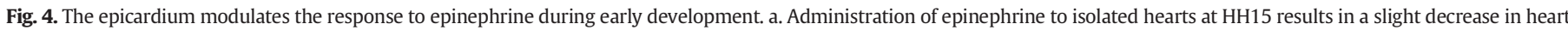

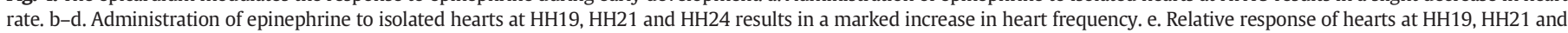
$\mathrm{HH} 24$ is significantly increased compared to the response of hearts at HH15. 

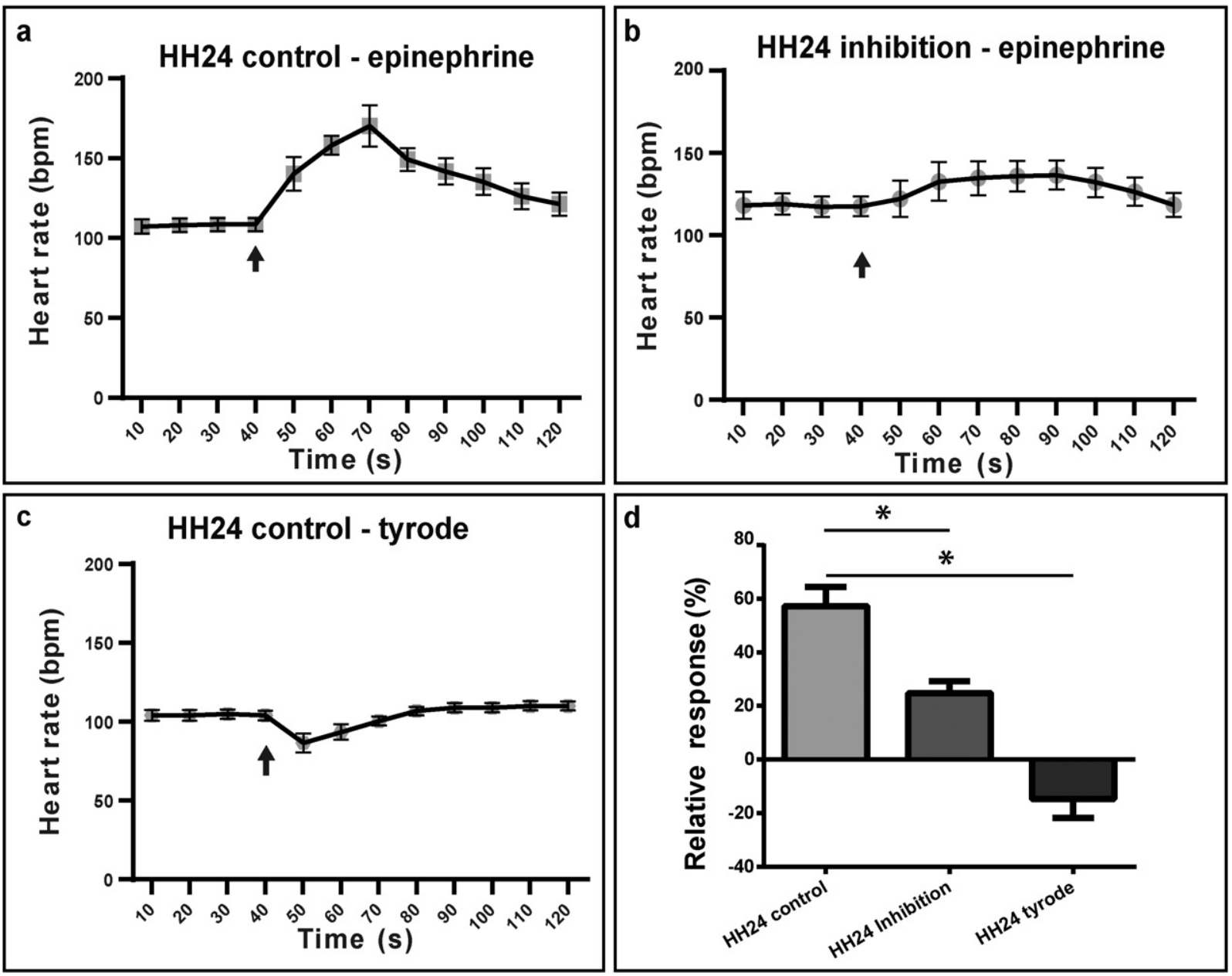

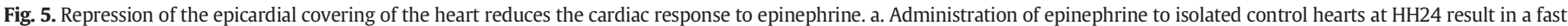

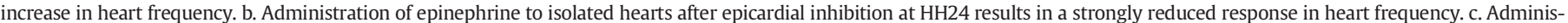

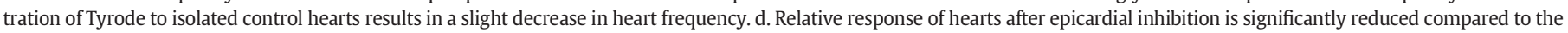
response of control hearts, ${ }^{*} \mathrm{p}=0,03$.

epicardial inhibition (or administration of teratogenic concentrations of all-trans RA [8]) could therefore result in aberrant $\beta 2 A R$ expression and an impaired response to its ligand, epinephrine.

Finally, recent work showed that normal development of the cardiac veins is required for normal development of the sympathetic nervous system of the heart [41]. This process is driven by nerve growth factor (NGF), secreted by vascular smooth muscle cells in subepicardial blood vessels [41]. Interestingly, the smooth muscle cells in the coronary vasculature derive from the epicardium after EMT $[42,43]$. Disturbing epicardial outgrowth results in abnormal development of the coronary vasculature [36]. The hampered response to epinephrine described in the current study could possibly be explained by disruption of the earliest stages of blood vessel formation and NGF production, which is required for normal development of the autonomic nervous system [41].

The current study has several limitations. It cannot be excluded that inhibition of the outgrowth of the epicardium has secondary effects on the heart which can affect cardiac functioning, since epicardial cells are important for differentiation and maturation of cardiomyocytes [44]. However, our results show that epicardial covering is important for the response to epinephrine, since normal hearts without epicardium (HH15) do not respond to epinephrine. However, as soon as cardiac outgrowth of epicardial cells has commenced (HH19), the heart shows a rapid response to epinephrine. The early response seen at HH19, makes it unlikely that myocardial differentiation is responsible for the change in heart rate seen after administration of epinephrine. Furthermore, the response seen in normal hearts at $\mathrm{HH} 15$ was comparable to the response seen in epicardially inhibited hearts. This again shows that it is not myocardial differentiation, but the presence of epicardium which is responsible for the response to epinephrine. Although the chicken model demonstrates highly reproducible results, the results described in the current work could possibly be strengthened by electrophysiological testing in a mammalian model, which shows defects in epicardial covering. However, up to date, there is no mouse model specifically affecting outgrowth of the epicardium, since the genes commonly used for epicardium-specific expression of Cre are known to be expressed more broadly throughout the fetus during development $[19,45]$.

In conclusion, the current study provides evidence indicating a role for the epicardium in autonomic modulation during early development. Autonomic modulation is essential for proper cardiac functioning and dysfunctioning of the autonomic nerve system is implicated in several diseases, such as cardiac arrhythmias, heart failure, congenital heart disease and hypertension [1-3]. The current study provides evidence indicating a role for the epicardium in autonomic modulation during early development. Further research is required to investigate the role of the epicardium in autonomic dysfunction seen in common cardiac disorders, and to explore the mechanisms responsible for the early heart rate response mediated by the epicardium. 

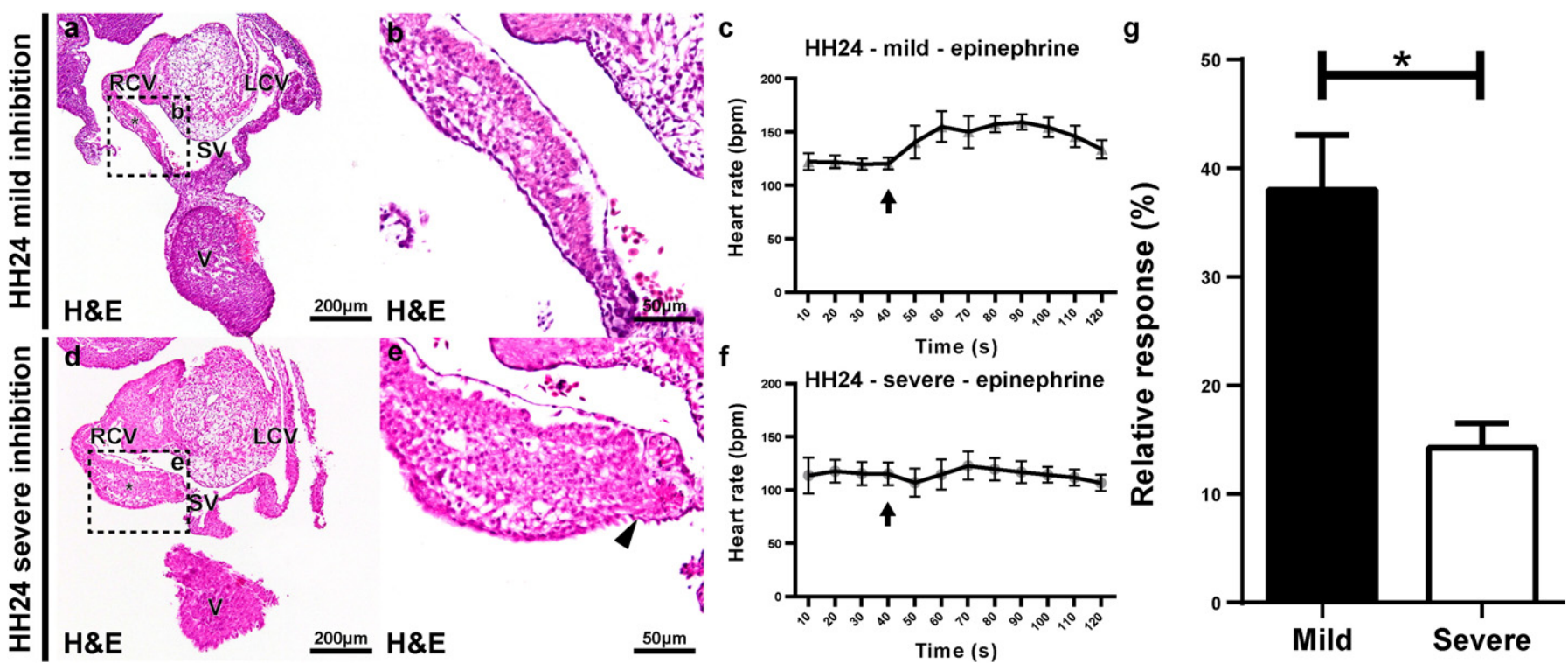

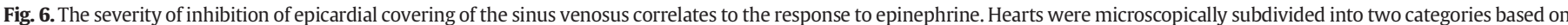

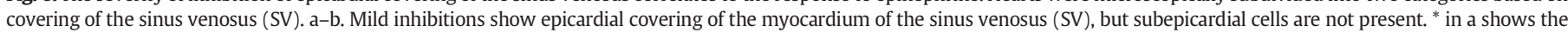

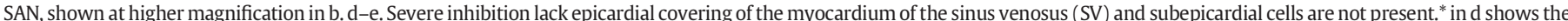

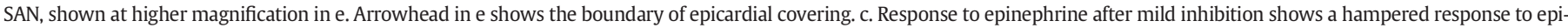

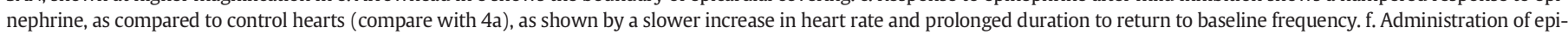

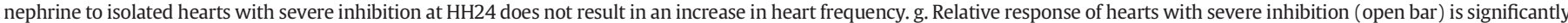

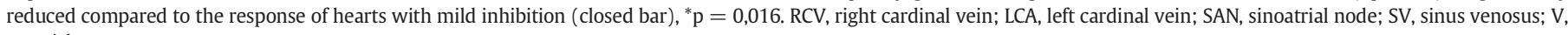
ventricle.

Supplementary data to this article can be found online at http://dx. doi.org/10.1016/j.yjmcc.2015.10.025.

\section{Disclosures}

None.

\section{Acknowledgments}

The authors thank L.J. Wisse and J.C. van Munsteren for their technical assistance.

This work was supported by grants from ZonMW/NWO, The Netherlands [90700368 to M.R.M.J. and 016.146.079 to A.M.S.], The Netherlands Heart Foundation, The Netherlands [2012T7.1 to RVS], a grant from the Dutch government to the Netherlands Institute for Regenerative Medicine [FES0908 to B.P.T.K.], Smartcare, part of the research program of the BioMedical Materials Institute, the Rembrandt Institute for Cardiovascular Disease and an LUMC fellowship [to A.M.S.].

\section{References}

[1] M.J. Shen, D.P. Zipes, Role of the autonomic nervous system in modulating cardiac arrhythmias, Circ. Res. 114 (2014) 1004-1021.

[2] G. Mancia, G. Grassi, The autonomic nervous system and hypertension, Circ. Res. 114 (2014) 1804-1814.

[3] V.G. Florea, J.N. Cohn, The autonomic nervous system and heart failure, Circ. Res. 114 (2014) 1815-1826.

[4] G.-P. Diller, et al., Heart rate response during exercise predicts survival in adults with congenital heart disease, J. Am. Coll. Cardiol. 48 (2006) 1250-1256.

[5] W. Hasan, Autonomic cardiac innervation: development and adult plasticity, Organogenesis 9 (2013) 176-193.

[6] V.Hildreth, R.H. Anderson, D.J. Henderson, Autonomic innervation of the developing heart: origins and function, Clin. Anat. 22 (2009) 36-46.

[7] M.E. Verberne, A.C. Gittenberger-De Groot, L. Van Iperen, R.E. Poelmann, Contribution of the cervical sympathetic ganglia to the innervation of the pharyngeal arch arteries and the heart in the chick embryo, Anat. Rec. 255 (1999) 407-419.

[8] J.M. Kroese, M.L.A. Broekhuizen, R.E. Poelmann, P.G.H. Mulder, J.W. Wladimiroff, Epinephrine affects hemodynamics of noninnervated normal and all-trans retinoic acid-treated embryonic chick hearts, Fetal Diagn. Ther. 19 (2004) 431-439.
[9] D.W. McGraw, S.B. Liggett, Molecular mechanisms of beta2-adrenergic receptor function and regulation, Proc. Am. Thorac. Soc. 2 (2005) 292-296 (discussion 311-312).

[10] S.N. Ebert, R.P. Thompson, Embryonic epinephrine synthesis in the rat heart before innervation: association with pacemaking and conduction tissue development, Circ. Res. 88 (2001) 117-124.

[11] S.N. Ebert, J.M. Baden, L.H. Mathers, B.J. Siddall, D.L. Wong, Expression of phenylethanolamine n-methyltransferase in the embryonic rat heart, J. Mol. Cell. Cardiol. 28 (1996) 1653-1658

[12] G. Teitelman, H. Baker, T.H. Joh, D.J. Reis, Appearance of catecholamine-synthesizing enzymes during development of rat sympathetic nervous system: possible role of tissue environment, Proc. Natl. Acad. Sci. U. S. A. 76 (1979) 509-513.

[13] V. Hamburger, H.L. Hamilton, A series of normal stages in the development of the chick embryo, J. Morphol. 88 (1951) 49-92.

[14] B. Ostádal, E.G. Krause, I. Beyerdörfer, V. Pelouch, A. Wollenberger, Effect of intraamnial administration of a cardiotoxic dose of isoproterenol on cyclic AMP levels in the chick embryo heart, J. Mol. Cell. Cardiol. 11 (1979) 1183-1187.

[15] R. Kockova, et al., Heart rate changes mediate the embryotoxic effect of antiarrhythmic drugs in the chick embryo, Am. J. Physiol. Heart Circ. Physiol. 304 (2013) H895-H902.

[16] L.L. Hoover, E.G. Burton, B.a. Brooks, S.W. Kubalak, The expanding role for retinoid signaling in heart development, Sci. World J. 8 (2008) 194-211.

[17] A.C. Gittenberger-de Groot, et al., The arterial and cardiac epicardium in development, disease and repair, Differentiation 84 (2012) 41-53.

[18] P. Carmeliet, et al., Impaired myocardial angiogenesis and ischemic cardiomyopathy in mice lacking the vascular endothelial growth factor isoforms VEGF164 and VEGF188, Nat. Med. 5 (1999) 495-502.

[19] S.N. Duim, K. Kurakula, M.-J. Goumans, B.P.T. Kruithof, Cardiac endothelial cells express Wilms' tumor-1, J. Mol. Cell. Cardiol. 81 (2015) 127-135.

[20] J. Männer, Experimental study on the formation of the epicardium in chick embryos, Anat. Embryol. (Berl.) 187 (1993) 281-289.

[21] R. Vicente-Steijn, et al., Electrical activation of sinus venosus myocardium and expression patterns of RhoA and Isl-1 in the chick embryo, J. Cardiovasc. Electrophysiol. 21 (2010) 1284-1292.

[22] M.P. Vrancken Peeters, M.M. Mentink, R.E. Poelmann, A.C. Gittenberger-de Groot Cytokeratins as a marker for epicardial formation in the quail embryo, Anat. Embryol. (Berl.) 191 (1995) 503-508.

[23] T.C. Katz, et al., Distinct compartments of the proepicardial organ give rise to coronary vascular endothelial cells, Dev. Cell 22 (2012) 639-650.

[24] M.A. Tischfield, et al., Human TUBB3 mutations perturb microtubule dynamics, kinesin interactions, and axon guidance, Cell 140 (2010) 74-87.

[25] H. Locher, et al., Class III $\beta$-tubulin, a novel biomarker in the human melanocyte lineage, Differentiation 85 (2013) 173-181.

[26] H. Locher, J.H.M. Frijns, M.A. Huisman, S.M. Chuva de Sousa Lopes, TUBB3: neuronal marker or melanocyte mimic? Cell Transplant. 23 (2014) 1471-1473.

[27] A. Von Gise, et al., WT1 regulates epicardial epithelial to mesenchymal transition through $\beta$-catenin and retinoic acid signaling pathways, Dev. Biol. 356 (2011) 421-431. 
[28] J.F. Armstrong, K. Pritchard-Jones, W.A. Bickmore, N.D. Hastie, J.B.L. Bard, The expression of the Wilms' tumour gene, WT1, in the developing mammalian embryo, Mech. Dev. 40 (1992) 85-97.

[29] R.R. Rackley, et al., Expression of the Wilms' tumor suppressor gene WT1 during mouse embryogenesis, Cell growth Differ. 4 (1993) 1023-1031.

[30] N. Wagner, et al., A splice variant of the Wilms' tumour suppressor Wt1 is required for normal development of the olfactory system, Development 132 (2005) 1327-1336.

[31] K.-D. Wagner, et al., The Wilms' tumor gene Wt1 is required for normal development of the retina, EMBO J. 21 (2002) 1398-1405.

[32] R. Parenti, et al., Immunolocalization of Wilms' tumor protein (WT1) in developing human peripheral sympathetic and gastroenteric nervous system, Acta Histochem. 116 (2014) 48-54.

[33] D. Greene, S. Kang, A. Kosenko, N. Hoshi, Adrenergic regulation of HCN4 channel requires protein association with $\beta 2$-adrenergic receptor, J. Biol. Chem. 287 (2012) 23690-23697.

[34] E. Merki, et al., Epicardial retinoid X receptor alpha is required for myocardial growth and coronary artery formation, Proc. Natl. Acad. Sci. U. S. A. 102 (2005) $18455-18460$.

[35] H. Lie-Venema, et al., Origin, fate, and function of epicardium-derived cells (EPDCs in normal and abnormal cardiac development, ScientificWorldJournal 7 (2007) 1777-1798.

[36] A.C. Gittenberger-de Groot, M.P. Vrancken Peeters, M. Bergwerff, M.M. Mentink, R.E. Poelmann, Epicardial outgrowth inhibition leads to compensatory mesothelial outflow tract collar and abnormal cardiac septation and coronary formation, Circ. Res. 87 (2000) 969-971.
[37] J.A. Kreidberg, et al., WT-1 is required for early kidney development, Cell 74 (1993) 679-691.

[38] J.A. Guadix, et al., Wt1 controls retinoic acid signalling in embryonic epicardium through transcriptional activation of Raldh2, Development 138 (2011) 1093-1097.

[39] C.M. Braitsch, M.D. Combs, S.E. Quaggin, K.E. Yutzey, Pod1/Tcf21 is regulated by retinoic acid signaling and inhibits differentiation of epicardium-derived cells into smooth muscle in the developing heart, Dev. Biol. 368 (2012) 345-357.

[40] M. Fennell, X.Z Khawaja, M.I. Cockett, A. Wood, Enhanced neuronal differentiation of NTera- 2 cells expressing neuronally restricted $\beta 2$ adrenergic receptor, Brain Res. 799 (1998) 243-249.

[41] J. Nam, et al., Coronary veins determine the pattern of sympathetic innervation in the developing heart, Development 140 (2013) 1475-1485.

[42] M.P.F.M. Vrancken Peeters, A.C. Gittenberger-de Groot, M.M.T. Mentink, R.E. Poelmann, Smooth muscle cells and fibroblasts of the coronary arteries derive from epithelial-mesenchymal transformation of the epicardium, Anat. Embryol. (Berl.) 199 (1999) 367-378.

[43] T. Mikawa, R.G. Gourdie, Pericardial mesoderm generates a population of coronary smooth muscle cells migrating into the heart along with ingrowth of the epicardial organ, Dev. Biol. 174 (1996) 221-232.

[44] A. Weeke-Klimp, et al., Epicardium-derived cells enhance proliferation, cellular maturation and alignment of cardiomyocytes, J. Mol. Cell. Cardiol. 49 (2010) 606-616.

[45] V.M. Christoffels, et al., Tbx18 and the fate of epicardial progenitors, Nature 458 (2009) E8-E9 (discussion E9-10). 